\title{
Improving Resistance against Attack of L2DCASKE Encryption Algorithm by using RCA Rule 30 based S-Box
}

\author{
K.J. Jegadish Kumar \\ Assistant Professor \\ SSN College of Engineering \\ Kalavakkam-603110 Chennai, \\ India
}

\author{
K. Chenna Kesava Reddy \\ Principal \\ T.K.R College of Engineering \\ and Technology \\ Meerpet-500078, Hyderabad, \\ India
}

\author{
S. Salivahanan \\ Principal \\ SSN College of Engineering \\ Kalavakkam-603110 \\ Chennai, India
}

\begin{abstract}
The proposed method L2D-CASKE, the lightweight 2dimensional (2-D) Cellular Automata (CA) based symmetric key encryption algorithm is a 128 bit length that allows a key length of 128 bits. It is designed as a lightweight encryption algorithm. As it is light weight, it can be easily applied on small devices such as wireless sensor motes, smart cards and other PDAs. An encryption algorithm having simple loop operations, based on iterative parameters is used in this work. A number of sequences and operations are used for this purpose. In this paper, an CA based S-Box is proposed for the L2DCASKE algorithm and the modified algorithm was verified using MATLAB 7.7 (R2008). To achieve this, the algorithm uses loop design based on iterations. Then the algorithm with the S-Box is tested for its strength compared to the conventional algorithm.
\end{abstract}

Keywords: Cellular automata, Symmetric Encryption, Substitution Permutation Network (SPN).

\section{INTRODUCTION}

New era of computing and communication technologies has initiated handling of different types of handy devices that are widely used for mobile applications. The portability and affordability factors of these devices are accomplished by imposing a limitation on its storage, power backup, and computational requirements. Regardless of the type of device used, secured communication is a prime requirement for every consumer using that device. Though many techniques exist in documentation for information security, this paper considered the cryptographic system based on symmetric key. Implementation of the standard algorithms requires a lot of computational time requirements, memory, area and power. The block ciphers realized on the smart card technology [1] experiences side channel attacks that depend on time and power. The procedures required to protect against such attacks are expensive and complex.

\subsection{Cellular Automata}

Cellular Automata (CA) is an organized grid of cells, and each cell has a finite number of states, such as "TRUE" (T) or "FALSE" (F). The grid dimensions can be of any finite value. Each cell within a group of cells is called hood. It is characterized relatively with respect to a particular cell. To start with at time $\mathrm{t}=0$, a state is assigned to the cells. The new states of the cell depend on its own previous state and states of its neighborhood. The new states are assigned based on some predefined rule using mathematical calculations. In the next time stamp, the cell is considered to be in state "T" when the neighborhood cells are also in state " $T$ ". If this condition is not satisfied, then the cell is in "F" state. Each cell in the lattice is simultaneously updated in this way with the prescribed rule sets and mathematical functions [2].

Encryption, by theory requires highly complex actions such as permuting, flipping and altering data in such a way that it is undecipherable and provides complex relationship with the original text and keys. This relationship should be non-linear so that decryption process is as tough as possible. The encryption process must be faster in time and cheaper in terms of the components involved [2]. CA provides a basic structure for highly parallel and complex operations upon which a basic encryption scheme can be built. CA based processor can be used to compute and modify data with a high degree of linearity and complexity [3].

The message encryption is done by Pseudo Random Number Generators (PRNGs) using CA. The generation of new states in One-Dimensional (1-D) CA can be considered as a sequence of random numbers [3]. Different security schemes have been proposed including symmetric key, hash functions and public key cryptography as observed by Sarkar, 2000. Further as, stated by Wolfram $[4,5]$, Rule 30 promotes the use of large integers in the pseudo random number generation. Owing to this fascinating chaotic property of the peculiar CA, Wolfram states that, this kind of $\mathrm{CA}$ is used as random number generator.

The structure of the paper is as follows: Section 2 describes symmetric key algorithms and S-Box design, Section 3 depicts the good cryptographic properties in detail and Section 4 illustrates Matlab implementation and its result analysis to test the functionality of the algorithm. Then basic cryptanalysis tests and its results were shown to prove the security level. Finally, conclusion is expressed in Section 5.

\section{ALGORITHM DESCRIPTION}

The entire L2DCASKE round comprises of simple XOR, Block Permutation, 1D RCA Key Generation, 2D RCA Margolus Neighborhood cell. The number of rounds ' $n_{r}$ ' values is chosen as 16. The RCA operator is used for 1-D CA based key generation. The detailed description of the algorithm is given in the reference [6]. Later, to the above algorithm additional operator called Substitution Box (S-Box) is added prior to the block permutation operation. This enhances the nonlinearity of the entire algorithm. 


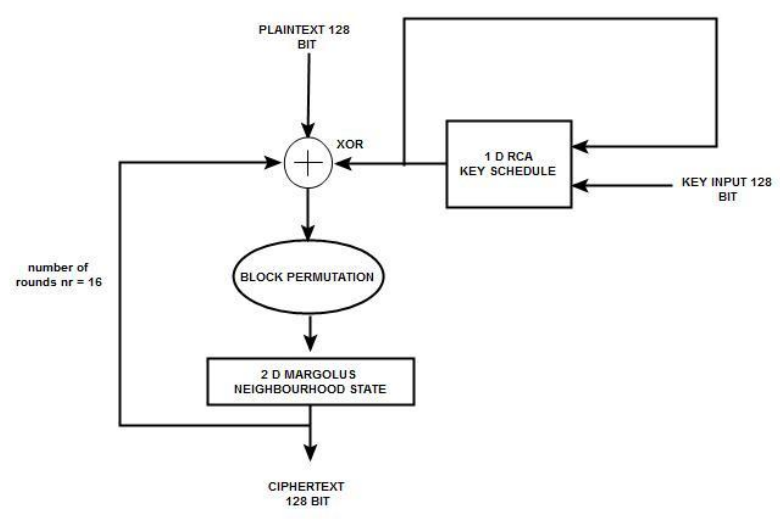

Figure 1: Proposed L2DCASKE Encryption Scheme without CA Based S-Box.

\subsection{D RCA Key Generation}

RCA is the one in which the preceding pattern can be recovered from the given current pattern(s). The proposed algorithm utilizes RCA (second order) [7]. The 'ith' state of $n$ bit pattern is determined by the clock cycles.

Given below is an example of a 3-neighborhood second order RCA [2]:

$$
\begin{aligned}
& x_{i}(t+1)=f\left(x_{i-1}(t), x_{i}(t), x_{i+1}(t)\right) \oplus x_{i}(t-1) ; \\
& x_{i}(t-1)=f\left(x_{i-1}(t), x_{i}(t), x_{i+1}(t)\right) \oplus x_{i}(t+1) .
\end{aligned}
$$

Here, the states $\mathrm{xi}(\mathrm{t}+1)$ and $\mathrm{x}_{\mathrm{i}}(\mathrm{t}-1)$ are denoted respectively by the terms $\xi_{\mathrm{i}}$ and $\mathrm{y}_{\mathrm{i} \text {. }}$. $\xi$ is obtained based on two initial patterns of $(\mathrm{Y}, \mathrm{X})$ at time steps $(\mathrm{t}-1)$ and $\mathrm{t}$. Then, using two successive patterns $(\xi, X)$, the initial pattern $Y$ can be figured out. This operation is denoted as follows [2].

$$
\xi=\operatorname{RCA}(\mathrm{Y}, \mathrm{X}) ; \mathrm{Y}=\operatorname{RCA}(\xi, \mathrm{X})
$$

The evolved pattern of such an RCA can be evaluated as [2]

$$
\mathrm{x}_{\mathrm{i}}(\mathrm{t}+1)=\left(\mathrm{x}_{\mathrm{i}-1}(\mathrm{t}) \oplus\left(\mathrm{x}_{\mathrm{i}}(\mathrm{t}) \vee \mathrm{x}_{\mathrm{i}+1}(\mathrm{t})\right) \oplus \mathrm{x}_{\mathrm{i}}(\mathrm{t}-1)\right) .
$$

One can refer Tripathy and Nandi for further details about RCA logic configurations [2]. As an example, states for the resultant configuration $\mathrm{Y}=\mathrm{RCA}(\mathrm{X}, \mathrm{K})$ using the rule 30 can be determined by the above equations.

\subsection{S-Box Design}

\section{Introduction to S-Box}

S-Box is the basic component of cipher system. It substitutes the value of input into another value as output. It can have a different number of inputs and outputs (m-bit input word and n-bit output word).
S-Box of two types

- Bijective: It is the S-Box in which the ' $\mathrm{m}$ ' input words are equal to ' $\mathrm{n}$ ' output words. $(m=n)$

- Non Bijective: It is the S-Box in which the ' $m$ ' input words are not equal to the ' $n$ ' output words. $(m \neq n)$.

A bijection (or bijective function are one to one correspondence) is a function giving an exact pairing of the elements of two sets [8]. Every element of one set is paired with exactly on the element of the other set, and every element of the other set is paired with exactly one element of the first set. Informal mathematical terms, a bijective function $\mathrm{f}: X \rightarrow Y$ is a one to one and onto mapping of a set $X$ to a set $Y$. A bijection from the set $X$ to the set $Y$ has inverse from $X$ to $Y$. If $X$ and $Y$ are finite sets, then the existence of a bijection means they have the same number of elements [8]. For infinite sets, the picture is more complex, leading to the concept of cardinal number, a way to distinguish the various sizes of infinite sets. A bijective function from a set to itself is also called a permutation [9].

Basically, there are two different types of design approaches as said below:

\section{Look Up Table (LUT) based.}

In the LUT based design, fixed table is normally used in which for each value of ' $m$ ' bit word, an alternate value of ' $n$ ' bit words is pre-defined. (Ex: S-Boxes in AES, DES.)

\section{Function based.}

In the function based design, the functions are defined such that

$$
f:\{0,1\}^{m} \rightarrow\{0,1\}^{n}
$$

\subsection{S-Box Using RCA Rule 30}

The Fig. 2 depicts the proposed single element of $8 \times 8$ RCA Rule 30 based S-Box. The single element of an 8 x 8 S-Box consists of eight inputs and eight outputs. According to the proposed cipher of 128 bit length, the plaintext is grouped into sixteen eight bit blocks. Hence, the modified L2DCASKE cipher system requires sixteen 8 x 8 S-Boxes.

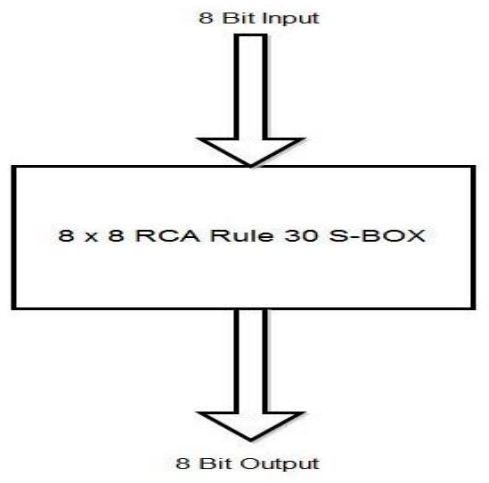

Figure 2. Single Element 8 x 8 RCA Rule 30 S-Box

The modified L2DCASKE using Substitution and Permutation Network (SPN) architecture is shown in Fig. 3 


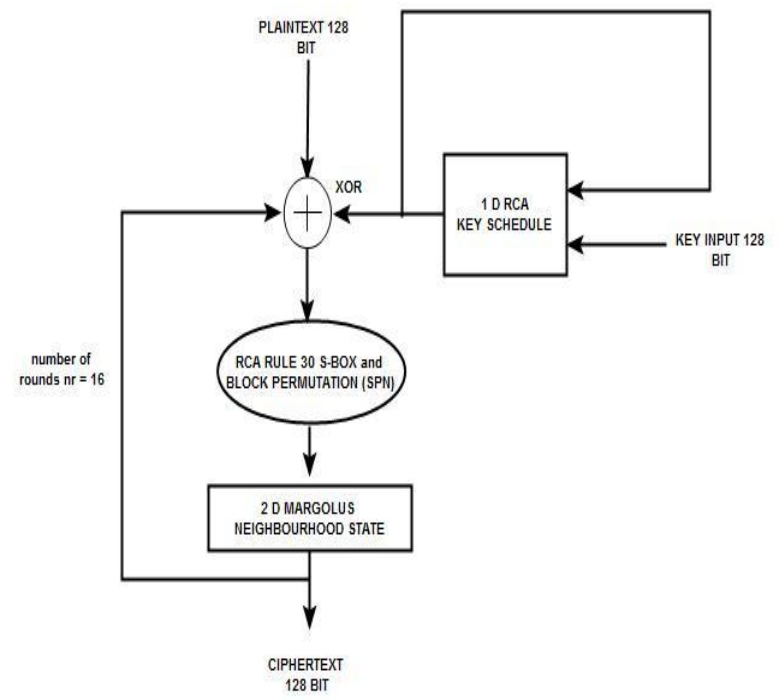

Figure 3. Modified L2DCASKE with S-Box

\subsection{Block Permutation}

The Block Permutation (BP) operation permutes each $2 \times 2$ sub-block within a $4 \times 4$ block diagonally. The idea behind this permutation is to move the data at the boundaries at the centre of the block and vice versa. This process results in enhanced diffusion rate $[6,9]$. So it is difficult to perform differential cryptanalysis. Further, through simple means of wire crossings, one can achieve hard-wiring which makes the implementation of block permutation as an easy process. The block permutation operation is described in Fig. 5. Increasing the redundancy content of the given plain-text is the principle idea of diffusion. The usage of Block Permutation operation in the proposed technique guarantees the diffusion condition.

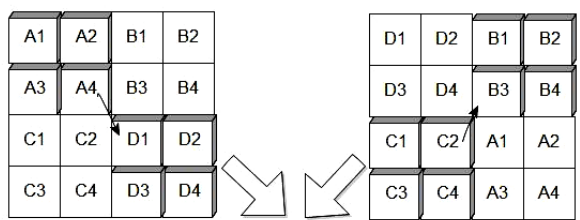

\begin{tabular}{|l|l|l|l|}
\hline D1 & D2 & C1 & C2 \\
\hline D3 & D4 & C3 & C4 \\
\hline B1 & B2 & A1 & A2 \\
\hline B3 & B4 & A3 & A4 \\
\hline
\end{tabular}

Figure 5. Block Permutation Operation

\subsection{D RCA: Margolus Neighborhood}

RCA in Two Dimensions: As we consider the plain-text to be of two-dimensions, we make use of 2D Reversible Cellular Automata (RCA). An approach called Partitioned Cellular Automata (PCA) is one more method to develop a reversible CA [11]. A familiar partitioning technique called Margolus neighborhood is selected for its inherent invertible property. Need for this arises from the fact that conventional CAs are not reversible and difficult to perform the inversion process.
The traditional Rule 30 evaluates the four likely patterns that stay alive for either state of the present cell. Evidently, there is a difficulty in determining the previous state of a cell with that of its present state. The main reason is that there is the likelihood of losing information while evaluation of the states of previous cell. Fig. 4(a) illustrates traditional 1-D CA [11] which consists of three inputs, one of them being the cell itself and other two being its neighbors. However, the output is simply one cell. However, the likelihood of this occurrence is not exceptionally high. Hence, we chose the Margolus neighborhood described in Fig. 4(b).
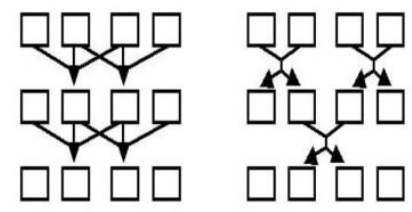

Figure 4: Conventional CA Vs Margolus Neighbourhood [19]

On the other hand, Margolus was interested in reversible CA [7, 11-13], for which rules encompassing shifting, reflection, creation, annihilation and complementation abound in such small neighborhoods; the Billiard-Ball Machine was a result. In general, partitioning schemes defined in book Cellular Automata Machines (CAM) by Norman Margolus called it as Margolus neighborhood. This simple partitioning scheme contains a grid of cells split into a set of four cells. In this Margolus neighborhood partitioning scheme, the CA is partitioned as displaced boxes containing 2 cells on the sides of each boxes. Then the partitioning alters from one clock cycle to the next cycle so that element of one box is associated as another element of an adjoining box on alternate clock cycles. In Margolus neighborhood, the contents of boxes are updated at once wholly as compared to the traditional 1-D CA neighborhood. The rule applied to the entire four cells to alter the states based D Rules, generally called as Billiard Ball Machine rules [7, 13]. In the Margolus neighbourhood, the entire matrix is divided into non-overlapping sub- blocks, each of $2 \times 2$ cells (bits), implying $2^{(2 \times 2)}=16$ possible configurations for every sub-block.

\section{CRITERIA FOR GOOD CRYPTOGRAPHIC ALGORITHMS}

A motivation for applying CA to realize S-boxes steams from potentially very attractive features of CA. CA are computationally universal (see e.g., [14], [15]), that means that such Boolean functions can be realized. Furthermore, CA of a given size and with their rules can potentially realize not one, but a number of S-box functions, that gives a possibility of designing much stronger cryptography systems. CA is a highly parallel system, easy in hardware implementation that results in high efficiency of CA-based cryptographic systems. The quality of S-boxes, also designed with the use of CA must be verified by required properties of S-boxes as nonlinearity, autocorrelation, balanced property and strict avalanche criterion. The most fundamental definitions and dependencies related to this issue are recalled from cryptographic literature [16], [17] and are given below. 


\section{A. Nonlinearity}

A Boolean function $f: B^{n} \rightarrow B$, maps $\mathrm{n}$ binary inputs to a single binary output. The list of the size of $2 \mathrm{n}$ of all possible outputs is the truth table. Polarity form of the truth table is denoted by and defined as $\hat{f}(x)=(-1) f(x)$. The nonlinearity $N_{f}$ of a Boolean function $\mathrm{f}$ is the minimal distance of the function $f$ to the set of affine functions and is calculated as given below [9]:

$$
N_{f}=\frac{1}{2}\left(2^{n}-W H_{\max }(f)\right) .
$$

Cipher systems with high non-linearity (low WHmax) are known to be more difficult to linear cryptanalysis (more secure).

\section{B. Autocorrelation}

The next important property of ciphers is autocorrelation. Autocorrelation Transform defines correlation between polar form and its polar shifted version. The absolute maximum value of any autocorrelation transform is defined as an autocorrelation and denoted by the equation [9]:

$$
A C_{f}=\max _{s \neq 0}\left|\sum_{x} \hat{f}(x) \hat{f}(x \otimes s)\right| .
$$

where $s \in B^{n} \rightarrow\{0\}$. Ciphers with low autocorrelation are known to be more secure.

\section{Balanced property}

Balance (regularity) is another essential criterion which should be fulfilled by a Boolean function used in ciphering. This means that each output bit (0 or 1) should appear an equally number of times for all possible values of inputs. The balance of a Boolean function is measured using its Hamming Weight and is defined as the equation [9]:

$$
H W=\frac{1}{2}\left(2^{n}-\sum_{x \in B^{n}} \hat{f}(x)\right) .
$$

Boolean function is balanced when its Hamming Weight is equal to $2^{\mathrm{n}}-1$. This gives resistance to differential cryptanalysis.

\section{Strict avalanche criteria (SAC)}

Strict Avalanche Criterion (SAC) was first introduced by Webster and Tavares. A Boolean function of $n$ variables satisfy SAC if complements of any of the $n$ input bits result in changing the output bit with probability equal to $1 / 2$. It means that for each of n-element vector $c^{n}$ with only one $\mathrm{i}^{\text {th }}$ bit of this vector equal to $1\left(c_{i}^{n}\right)$, the following equation is satisfied [9]:

$$
\sum_{x \in B^{n}} f(x) \oplus f\left(x \oplus c_{i}^{n}\right)=2^{n-1}
$$

The analysis of satisfaction of SAC for Boolean function $f$ is measured by the distance, $d S A C_{f}$, which is expressed by the equation [9]:

$$
d S A C_{f}=\max _{1 \leq i \leq n}\left|2^{n-1}-\sum f(x) \oplus f\left(x \oplus c_{i}^{n}\right)\right| .
$$

One can see that for ideally balanced a Boolean function $\mathrm{f}$ the value of $d S A C_{f}$ is equal to 0 . As the function which is not ideally balanced, the values of $d S A C_{f}$ will be in the range $\left(0,2^{\mathrm{n}}-1\right]$.

\section{IMPLEMENTATION OF ALGORITHM AND RESULT ANALYSIS}

The objective of designing and implementing a 2D CA-based symmetric-key encryption algorithm has been fulfilled. MATLAB was used as the development and testing platform. The necessary criteria for a strong cipher is that the relationship between the plain-text, the key and the cipher-text should be as involved and complex as possible, making it almost impossible to decipher the plain-text from the ciphertext without the exact key. The cipher-text on deciphering with the exact key should yield the plain-text without any error. Various tests were performed on the proposed algorithm to ensure that both the above criteria are satisfied. Plain texts of size 128-bits and key length of 128 bits were chosen and encrypted using the proposed cipher. The cipher text, the decrypted data and the difference between the plain-text and the cipher-text were obtained corresponding to each plaintext.

\section{A. Basic Test Results}

The results of some basic Tests and statistical analysis done for 1000 Random data such as Non-linearity, Balanced property, Autocorrelation, Variance and Standard deviation of Nonlinearity test and in cipher-text with respect to the plaintext, etc. are summarized below in table $1 \& 2$. The test decision for $n=128$, satisfying the criteria in the table 1 is such as for the better nonlinearity the calculated value should be equal to maximum value 64 . From the table, it is clearly understood the modified L2DCASKE has the value greater than the conventional proposed design, also proves to have better balanced property( Hamming weight should be equal to 64) and autocorrelation( Should have the lowest value as possible).

Table 1: Test Results for 1000128 Bit random data

\begin{tabular}{|c|c|c|}
\hline Test Criteria & $\begin{array}{c}\text { L2DCASKE } \\
\text { without S-Box }\end{array}$ & $\begin{array}{c}\text { L2DCASKE with } \\
\text { S-Box }\end{array}$ \\
\hline Non-linearity & 56.485 & 58.367 \\
\hline $\begin{array}{c}\text { Balanced } \\
\text { Property }\end{array}$ & 63 & 64 \\
\hline $\begin{array}{c}\text { Autocorr- } \\
\text { elation }\end{array}$ & 10.125 & 8.3750 \\
\hline
\end{tabular}


Table 2: Statistical Test Analysis

\begin{tabular}{|c|c|c|}
\hline Statistical Tests & $\begin{array}{c}\text { L2DCASKE } \\
\text { without S-Box }\end{array}$ & $\begin{array}{c}\text { L2DCASKE with } \\
\text { S-Box }\end{array}$ \\
\hline $\begin{array}{c}\text { Variance of Non- } \\
\text { linearity }\end{array}$ & 2.2750 & 1.7984 \\
\hline $\begin{array}{c}\text { Variance of } \\
\text { Balanced } \\
\text { Property }\end{array}$ & 32.41 & 28.189 \\
\hline
\end{tabular}

\section{CONCLUSION}

A two dimensional Cellular Automata based light-weight symmetric-key cryptosystem with an S-Box for hardware cost effective applications is proposed in this work. This encryption scheme meets all the basic requirements met out by the NIST standards. Also, this being light-weight, it can be easily applied on small devices such as sensor motes, smart cards, etc. The basic automata can be down scaled to work even on a basic 8-bit processor. The major advantage of the proposed scheme is the use of dynamic key schedule which minimizes the memory requirement on the smaller devices.

\section{REFERENCES}

[1] P. Kocher, J. Jaffre and B. Jun, "Differential power analysis," Crypto'99, LNCS 1666, pp. 398-412, Springer- Verlag, 1999.

[2] S Tripathy and S Nandi, "LCASE: Lightweight Cellular Automata-based Symmetric-key Encryption", International Journal of Network Security, Vol.8, No.2, Mar. 2009.

[3] Palash Sarkar, "A Brief History of Cellular automata", Journal of ACM Computing Surveys (CSUR), Volume 32 Issue 1, March 2000.

[4] S. Wolfram, "Cryptography with Cellular Automata," Crypto '85, LNCS 218, pp. 429- 432, Springer-Verlag, 1986.

[5] S. Wolfram, "Random sequence generation by cellular automata," Advances in Applied Maths, vol. 7, No. 2, pp. 123-169, 1986.
[6] K. J. Jegadish Kumar, K. Chenna Kesava Reddy, S. Salivahanan, "Novel and Efficient cellular automata based symmetric key encryption algorithm for Wireless Sensor Networks," International Journal of Computer Applications, Vol.23, No.4, June 2011.

[7] Weinberg, Steven. "Is the Universe a Computer?". (October 24, 2002), The New York Review of Books (Rea S. Hederman). Retrieved October 12, 2012.

[8] http://en.wikipedia.org/wiki/Bijection.html

[9] Szaban. M, "CA-based generator of S-boxes for cryptography use", Parallel \& Distributed Processing, Workshops and Phd Forum (IPDPSW), 2010 IEEE International Symposium, April 2010, pp 1-8.

[10] "Cellular Automata Rules Lexicon - Margolus Neighborhood." Free Software of mirek Wojtowicz. http://www.mirekw.com/ca/rullex_marg.html.

[11] "2D Reversible Cellular Automata" Lotus Artificial Life.http://www.alife.co.uk/ca/bbm/2d/.

[12] Joaquin Cerda, Rafael Gadea, and Guillermo Paya, "Implementing a Margolus Neighborhood Cellular automaton FPGA", IWANN 2003, LNCS 2687, pp. 121-128, Springer-Verlag Berlin Heidelberg, 2003.

[13] Jérôme Durand-Lose "Representing Reversible Cellular Automata with Reversible Block Cellular Automata", Discrete Mathematics and Theoretical Computer Science Proceedings AA (DM-CCG), 2001, pp 145-154.

[14] Adams. C, Tavares. S, "Good S-boxes are easy to find", Advance in cryptology, Proc. Of CRYPTO'89, LNCS 435, 1990, pp $612-615$.

[15] Clark. J. A, Jacob. J. L, Stepney. S, "The Design of SBoxes by Simulated Annealing, New Generation Computing", Vol. 23, No. 3, Ohmsha and Springer 2005, pp $219-231$.

[16] Albert. J, Culik. K II, "A simple universal cellular automaton and its one-way and totalising version", Complex Systems, Vol. 1, 1987, pp 1 - 16.

[17] Smith. A. R III, "Simple computation-universal cellular spaces”, Journal ACM, Vol. 18, 1971, pp 339 - 353. 